'Fundação Oswaldo Cruz (Fiocruz), Instituto René Rachou - Belo Horizonte (MG), Brasil.

Orcid: https://orcid. org/0000-0002-33846657

romulo.paes@minas.

fiocruz.br

2 Universidade Federal da Bahia (UFBA), Instituto de Saúde Coletiva (ISC)

- Salvador (BA), Brasil. Imperial College London, Department of Primary Care and Public Health

Public Health Policy

Evaluation Unit - Londres, Inglaterra. Fundação Oswaldo Cruz (Fiocruz),

Centro de Pesquisas Gonçalo Moniz - Salvador (BA), Brasil. Orcid: https://orcid org/0000-0002-72604386

davide.rasella@gmail.com

3 Pesquisador autônomo

- Belo Horizonte (MG),

Brasil.

Orcid: https://orcid org/0000-0002-7195-

8094

jcarepa@gmail.com

\section{Política econômica e saúde pública: equilíbrio fiscal e bem-estar da população}

\author{
Economic policy and public health: fiscal balance and population \\ wellbeing
}

Rômulo Paes-Sousa', Davide Rasella², Julio Carepa-Sousa ${ }^{\mathbf{3}}$

DOI: 10.1590/0103-11042018S313

RESUMO Muitos estudos examinaram os efeitos das crises econômicas e políticas de austeridade em países de renda alta. Contudo, são muito recentes e esparsos os estudos sobre os efeitos sobre países de renda média e baixa. Neste ensaio, revisitam-se os estudos recentes, procurando destacar o que pode ter incidência mais imediata sobre a saúde pública, com especial interesse sobre os grupos de maior vulnerabilidade. Optou-se por tratar de forma conjunta os efeitos da crise econômica e da política de austeridade percorrida até o presente momento no Brasil por entender que os deficit de investimento em políticas sociais e suas implicações sobre a cobertura e sobre qualidade dos serviços ofertados estão associados aos dois fenômenos. As publicações recentes indicam a piora de indicadores básicos de saúde nacionais, sugerindo que a redução de investimentos em saúde já se faz sentir nas políticas de promoção, prevenção e atenção. O debate econômico atual apresenta erroneamente as medidas de austeridade como único caminho para um regime de responsabilidade fiscal. O País precisa rever sua rota de desenvolvimento não apenas para enfrentar os efeitos da crise econômica em curso, mas também para recuperar sua trajetória de inclusão social e econômica e de melhora da saúde de sua população.

PALAVRAS-CHAVE Modelos econômicos. Qualidade de vida. Estratégia Saúde da Família. Proteção social.

ABSTRACT Many studies have observed the effects of economic crises and austerity policies on high income countries. However, few and very recent ones start to cover the effects on low and medium income countries. In this perspective, we revise the recent studies focusing on the immediate effects on public health, specially on the most vulnerable population. We preferred to consider the joint effects of the economic crises and austerity for understanding that the funding deficits of social policies and their implications on coverage and quality of service provision are interlinked. Recent publications indicate the decline of basic public health indicators, suggesting that the reduction of investments can already be felt on policies for health promotion, prevention and care. The currently economic debate falsely presents austerity measures as the single alternative for promoting fiscal responsibility. Brazil needs to change its development route not only to fight the effects of the economic crises but also to recover the way for improvement on socioeconomic inclusion and public health.

KEYWORDS Economic models. Quality of life. Family Health Strategy. Social protection. 


\section{Introdução}

Muitos estudos examinaram os efeitos das crises econômicas e políticas de austeridade em países de renda alta, contudo, são muito recentes e esparsos os estudos sobre os efeitos sobre países de renda média e baixa. No caso brasileiro, estudos recém-publicados alertam para a possível rápida degradação dos serviços públicos de saúde no País e seus efeitos deletérios sobre os indicadores de saúde da população'. Cabe especial atenção aos efeitos sobre a população infantil, particularmente as crianças que pertencem a famílias pobres e residem em municípios cuja maioria da população é constituída de pobres ${ }^{2}$.

Conforme o que foi descrito em outros artigos deste número especial, o Brasil apresentou um quadro de recessão econômica nos anos de 2015 e 2016, produzindo recuperação tímida em 2017. Estima-se que o baixo crescimento deve se prolongar até 2020, ano este em que, provavelmente, o País deverá retornar ao patamar de crescimento de $2014^{3}$, sendo, todavia, incapaz de repor as perdas do período recessivo. A Emenda Constitucional 95 foi produzida como resposta à crise em 2016, sendo implementada a partir de 2017 , e tendo como eixo articulador a supressão o piso mínimo de investimento social, conforme previsto na Constituição de 1988, e limitação do aumento de gastos públicos aos níveis de inflação4. Tais iniciativas tendem a comprometer o já deficitário orçamento da saúde pública no Brasil.

Neste ensaio, revisitamos os estudos recentes procurando destacar o que pode ter incidência mais imediata sobre a saúde pública, com especial interesse sobre os grupos de maior vulnerabilidade. Optamos por tratar os efeitos da crise econômica e da política de austeridade percorrida até o presente momento no Brasil por entendermos que os deficit de investimento em políticas sociais e suas implicações sobre a cobertura e qualidade dos serviços ofertados estão associados aos dois fenômenos ${ }^{5}$.

\section{Os impactos da crise econômica sobre a saúde dos países de renda alta}

Os efeitos da crise econômica e das políticas de austeridade fiscal sobre a saúde dos países de renda alta foram extensamente estudados pela comunidade científica. Schramm et al. ${ }^{5}$ reviram 885 estudos cobrindo os efeitos sobre os riscos à saúde, sobre o perfil epidemiológico e sobre os sistemas e serviços de saúde no período de 2006 a 2017. Os estudos cobrem principalmente os países da Organização para a Cooperação e Desenvolvimento Econômico (OCDE) e os demais países europeus que não pertencem a esta Organização. Os países não abordados no âmbito do artigo de Schramm et al. ${ }^{5}$ aparecem em publicações esparsas.

Alguns achados desta revisão são fundamentais para a compreensão do contexto brasileiro. Reeves et al.6, ao analisarem os efeitos das políticas de austeridade sobre o gasto em saúde de 27 países europeus, observaram que o orçamento da saúde não foi preservado nas crises econômicas. Os autores também assinalaram que os cortes não são consequências inexoráveis de: recessões, mudança anual do Produto Interno Bruto (PIB) per capita, perdas cumulativas no PIB. Curiosamente, eles não encontraram correlação entre a ideologia dos partidos governantes e os cortes em saúde, indicando que partidos ou coalisões tanto de esquerda quanto de direita lançaram mão de medidas de austeridade no enfrentamento da crise. Também, partidos de orientação ideológica oposta optaram por medidas de desenvolvimento no enfrentamento de seus desafios nacionais. Ainda no estudo de Reeves et al.6, foi encontrada correlação entre o aumento da dívida pública e cortes no gasto em saúde, havendo cortes maiores entre os que fizeram acordo com o Fundo Monetário Internacional. Ademais, os autores destacam que países adeptos do sistema de seguro social foram 
menos suscetíveis às políticas de austeridade, mas foram mais suscetíveis à queda do PIB per capita no período de 1995 a $2011^{6}$.

Os efeitos da crise econômica sobre a Grécia, pela sua contundência e confluência com a crise política, apresentaram fortes impactos sobre a saúde pública. $\mathrm{Em}$ uma revisão sobre os estudos publicados sobre esse país entre 2009 e 2013, Simou e Koutsogeorgou ${ }^{7}$ observaram a redução do gasto público em saúde, tanto na provisão de serviços quanto em gestão, ocorrendo ainda importantes efeitos sobre a força de trabalho da saúde. Para esse grupo de trabalhadores, foi observado: extinção de postos de trabalho com demissão dos titulares, redução da carga horária, além de perdas salariais e previdenciárias importantes. No tocante à infraestrutura, as autoras verificaram a redução na oferta de serviços de saúde, incluindo os serviços providos por hospitais universitários. Há registros, inclusive, de fechamento de equipamentos de saúde. Em relação à produção de fármacos, as autoras observaram o aumento do consumo no início do período observado - sobretudo de medicamentos para o tratamento de doenças psíquicas -, seguido pelo declínio do consumo em função da ausência de recursos financeiros para sua aquisição pelo Estado e pelos indivíduos, que levou ao fechamento de fábricas de medicamentos no país. Na Grécia, conforme as autoras, também ocorreu a redução no financiamento de pesquisa biomédica ${ }^{7}$.

Em relação ao perfil epidemiológico que emergiu em consequência da crise e da austeridade, Schramm et al. ${ }^{5}$ encontraram: transtornos mentais (suicídio, tentativas de suicídios e depressão), doenças infecciosas (HIV, Tuberculose, Malária e Febre do Nilo), Doenças Crônicas Não Transmissíveis (Doenças cardiovasculares) e Doenças da infância (asma e acidentes domésticos). Tratase de um perfil compatível ao crescimento da pobreza, aumento de exposição a riscos de infecção, e ao estresse vinculado à escassez de renda proveniente do trabalho ou das transferências sociais. No tocante aos fatores sociais, os autores identificaram estudos que dão conta ainda dos efeitos sobre a saúde do desemprego, precarização do trabalho, perda do seguro saúde, medo da perda do emprego e suas consequências, perda da moradia ou redução de qualidade da moradia, assim como redução do consumo de alimentos. Segundo os autores, alcoolismo e tabagismo apresentaram resultados divergentes, indicando que o consumo poderia ser estimulado pela dinâmica da crise e, ao mesmo tempo, ser refreado pela ausência de recursos para sua aquisição.

Schramm et al. 5 , ao analisarem os sistemas de saúde segundo uma tipologia de financiamento da saúde, encontraram referências à redução expressiva do gasto em saúde. O setor saúde foi afetado nos países que dispõem de sistema nacional de saúde, como Reino Unido e Suécia; de seguro social, como Alemanha e Japão; e de seguro privado, como Estados Unidos e Austrália. Nos Estados Unidos, observaram significativa redução do acesso aos serviços de saúde em função do desemprego e consequente redução do seguro saúde. Também nos Estados Unidos, observaram a redução das doações aos serviços de medicina filantrópica. De maneira geral, a literatura revista indicou o impacto da redução de financiamento na promoção, prevenção e atenção em saúde. Tanto os investimentos em pesquisa e desenvolvimento como em gestão foram afetados em decorrência de uma redução de gastos. Dessa forma, os estudos indicam a redução na rede prestadora de serviços em vários países, associados à redução dos recursos humanos e dos insumos. Os efeitos da escassez por seu turno tiveram repercussão sobre a qualidade dos serviços prestados, afetando a capacidade administrativa, gerencial e, ainda, a qualidade dos serviços prestados. No âmbito intradomiciliar, a combinação da redução da renda do trabalho com a redução das transferências sociais afetou os hábitos e atitudes das populações mais pobres em sua relação 
com riscos e cuidados de saúde. As alterações quanto à dieta e ao aumento do estresse foram registradas em muitos países. Já o tabagismo e o uso abusivo de álcool foram afetados de forma diversa: ora reduzidos devido à perda do poder aquisitivo, ora aumentados como resposta à expansão das tensões decorrentes do agravamento da pobreza familiar. A redução ou iminente redução do poder aquisitivo alterou a busca de serviços de saúde, sendo o adiamento ou a supressão da busca desses serviços pela redução dos gastos familiares ou como forma de evitar o estigma de portador de saúde débil e, portanto, vulnerável a ser incluído no grupo dos prioritários em caso de demissão 5 .

\section{Crise e austeridade no Brasil}

A crise econômica enfrentada pelo Brasil já acumula alguns anos, e suas consequências revelam um quadro crítico. Entre os indicadores que mais claramente ilustram tal situação, podemos citar os números de pobreza e extrema pobreza no País. A série histórica divulgada pelo Instituto Brasileiro de Geografia e Estatística (IBGE), que começa em 1992 utilizando dados da Pesquisa Nacional por Amostra de Domicílios (PNAD), apresenta expressiva redução a partir de 2003, quando 41,8 milhões e 12,9 milhões de pessoas se encontravam em situações de pobreza e extrema pobreza, respectivamente. $\mathrm{O}$ indicador de pobreza alcançou seu menor nível em 2014, quando 14,1 milhões estavam nessa situação, enquanto a pobreza extrema estava no segundo menor nível da história com 5,2 milhões de pessoas. Como reflexo dos efeitos da crise e das políticas de austeridade, ambos os indicadores foram significativamente impactados. Enquanto mais de 20 milhões se encontravam em situação de pobreza ao final de 2017, alarmantes 11,8 milhões de pessoas estavam em situação de pobreza extrema, valor não captado pelas PNAD desde $2004^{8}$.
A desigualdade de renda aferida pelo Índice de Gini vem apresentando crescimentos sucessivos desde abril de 2014, saltando de 0,5636 nesse mês para 0,5915 em fevereiro de $2018^{9}$. Dessa forma, o Brasil de 2018 apresenta uma distribuição de renda mais desigual que o mesmo país há quatro anos.

Além dos impactos imediatamente identificáveis, existem as consequências de longo prazo igualmente significativas. Uma dimensão relevante e complementar à análise de renda é a mobilidade social, que sugere o desafio enfrentado para que filhos de famílias de baixa renda possam ascender a patamares de renda menos desfavorecidos. Dados da OCDE sobre o desafio de transitar da baixa para a média renda indicam, no País, o segundo mais longo desafio de quebra desse ciclo intergeracional de pobreza, empatado com a África do Sul e atrás apenas da Colômbia ${ }^{10}$. No Brasil, são esperadas, em média, nove gerações para que a condição de renda baixa seja superada, evidenciando as dificuldades enfrentadas na tentativa de ascensão social. O dado indica que os impactos no arrefecimento do combate à pobreza terão efeito em inúmeras gerações vindouras; consequentemente, o agravamento no quadro de saúde da população de maior vulnerabilidade tende a perdurar ainda mais em decorrência da dinâmica social brasileira.

Corroboram para essa leitura as evidências apresentadas por Vieira e Benevides 4 que, em uma revisão de estudos sobre a crise nos países de alta renda e suas lições para o Brasil, concluíram que as crises econômicas podem agravar os problemas sociais e aumentar as desigualdades, levando à piora da situação de saúde da população. Já as medidas de austeridade fiscal, que estabelecem a redução do gasto com programas de proteção social, tendem a agravar os efeitos da crise sobre a situação de saúde, em particular, e as condições sociais, de forma mais geral. Diante disso, os autores recomendam a preservação dos programas de proteção social como medida importante 
para proteção da saúde da população e para a retomada do crescimento econômico em prazo mais curto.

Schramm et al. ${ }^{5}$ especulam que, em países de modelo híbrido de saúde como o Brasil, com forte presença dos setores público e privado, a redução do acesso aos bens e serviços pode ocorrer em vários trajetos simultâneos. O setor público pode ser pressionado pelo aumento da demanda de populações que, até o período anterior à crise, eram atendidas pelo setor privado, porém tiveram parte desse acesso revogado em decorrência da redução da atividade econômica e aumento do desemprego justamente quando o próprio setor público enfrenta uma grande retração no seu financiamento e maior dificuldade para oferecer a cobertura necessária.

As evidências dão conta de que a tentativa de equilíbrio das contas públicas no País resultou em medidas de austeridade com severa redução da rede de proteção social. Os gastos totais com saúde em 2017 foram praticamente os mesmos que os realizados em 2016, ainda que o Índice Nacional de Preços ao Consumidor Amplo (IPCA) de 2016 tenha registrado alta nos preços de $6,29 \%$. O orçamento total para a saúde previa gastos de $\mathrm{R} \$$ 114,7 bilhões, e o piso constitucional indicava que deveriam ser aplicados ao menos R $\$ 109$ bilhões". Contudo, a aplicação efetiva dos recursos ficou aquém de ambas as referências, totalizando $\mathrm{R} \$ 107,2$ bilhões e registrando, pela primeira vez, gastos abaixo do piso constitucional ${ }^{10}$. Morosini et al. ${ }^{12}$ apontam que alterações realizadas em 30 de agosto 2017 na Política Nacional de Atenção Básica tendem a enfraquecer esse nível de atenção quanto a: cobertura universal, acesso, equipe profissional, organização do processo de trabalho e coordenação nacional da política. Para as autoras, a flexibilização pretendida no modelo em um contexto de escassez de recursos levará ao rebaixamento do volume e da qualidade dos serviços prestados.

Inúmeros outros programas sociais também enfrentaram cortes expressivos. Em 2017, foram excluídas do Bolsa Família (BF) mais de 1 milhão de famílias, além da redução do escopo de programas como Brasil Sem Miséria e expressivos cortes orçamentários em outras iniciativas de proteção social como o Programa Cisternas e o Programa de Aquisição de Alimentos, que enfrentaram reduções de 90\% e 99\%, respectivamente ${ }^{\mathbf{1 0}}$. Ainda que não seja possível traçar uma correlação direta entre as medidas e algumas consequências verificadas recentemente, acontecimentos, como o recente surto de sarampo ${ }^{13}$, sugerem forte impacto negativo pelo enfraquecimento da rede pública de saúde.

Com o intuito de avaliar em maior profundidade os impactos de longo prazo dessa das medidas de austeridade, Rasella et al. ${ }^{2}$ buscaram avaliar o impacto da redução dos gastos públicos nas Metas de Desenvolvimento Sustentável por meio dos indicadores de saúde. Tendo em vista o longo alcance da Emenda Constitucional 95, que visa limitar os gastos públicos por um horizonte temporal de 20 anos, os autores buscaram avaliar o efeito esperado na mortalidade e hospitalização de crianças de até 5 anos entre 2017 e 2030 (data final para apuração das metas de Desenvolvimento Sustentável estabelecidas pela Organização das Nações Unidas - ONU).

Os programas com grande probabilidade de sofrer contração pelas medidas de austeridade econômica são o BF e a Estratégia Saúde da Família (ESF). Estudos sugerem que ambos os programas tiveram impactos positivos na redução da mortalidade de crianças e desigualdades na saúde, contribuindo para o alcance de uma das metas do Objetivo 3 de Desenvolvimento do Milênio (redução de dois terços da mortalidade de crianças de até 5 anos de idade).

A metodologia adotada no estudo implicou um levantamento da população nos municípios brasileiros; e, a partir de análises de impacto previamente conduzidas nas populações de 2000 e 2010, foram estimadas probabilidades associadas ao impacto de programas de redução da pobreza. A extrapolação dessas probabilidades foi usada 
para, a partir de 2010, mensurar o impacto dos programas BF e Saúde da Família, com seus respectivos orçamentos ajustados pelas medidas de austeridade, na população estimada de idade inferior a 5 anos até 2030, obedecendo a dinâmica populacional projetada para o Brasil nesse período ${ }^{2}$.

Foram simulados três cenários de crise econômica utilizando taxas de pobreza e renda per capita média a partir da PNAD para os anos de 2011 a 2015 e estimativas do Banco Mundial: no cenário 1, uma crise mais amena, com aumento na taxa de pobreza de 0,55\% durante 3 anos (2015 a 2017); o cenário 2 estima uma crise média com aumento de $0,8 \%$ na taxa de pobreza por 5 anos (2015 a 2019); o cenário 3 estima aumento da taxa de pobreza de $0,8 \%$ por um prazo total de 7 anos (2015 a 2021). O cenário 2 é aquele considerado mais provável pelos autores, portanto, para fins deste ensaio, a análise será concentrada no caso de crise econômica intermediário, para o qual os resultados de Austeridade, Saúde e Proteção Social são apresentados em seguida ${ }^{2}$.

Como resposta à crise econômica, dois cenários de políticas foram simulados. Austeridade fiscal: iniciado a partir de 2017. Estimativas foram baseadas em simulações de impacto de medidas de austeridade já implementadas (principalmente em decorrência da EC 95) nos orçamentos de proteção social e atenção à saúde até $2030^{4,14,15}$. Reduções nas coberturas do BF e da ESF proporcionais à redução do orçamento. Proteção Social: manutenção da cobertura de assistência social e atendimento de saúde em resposta à crise no período entre 2017 e 2030. Essa resposta foi projetada como incremento da cobertura de BF e ESF proporcional ao aumento da taxa de pobreza durante $o$ período de crise, seguida de redução no BF proporcional à redução à pobreza e retorno do ESF a níveis pré-crise ${ }^{2}$.

Gráfico 1. As taxas médicas de mortalidade infantil de menores de cinco anos (por mil nascidos vivos) sob uma crise econômica intermediária e cenários de resposta de políticas para 2010-2030

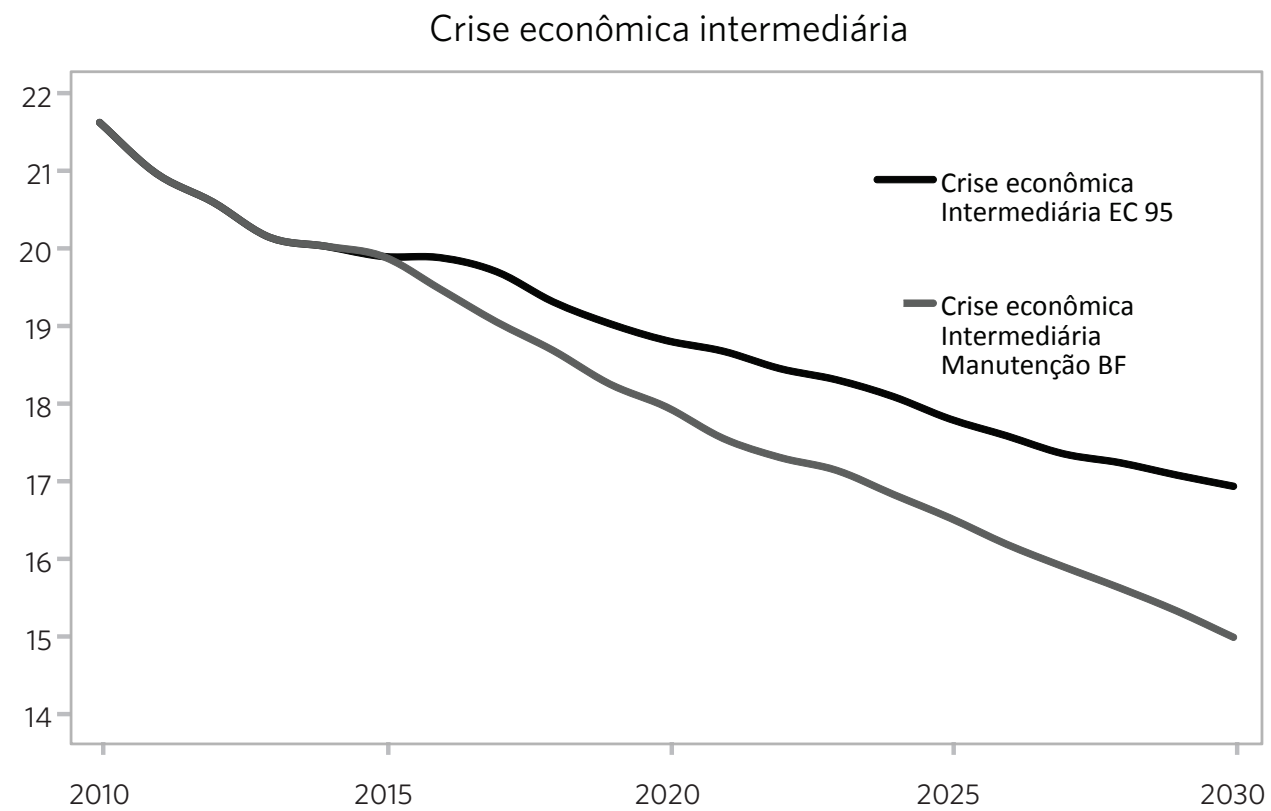

Fonte: Rasella D, Basu S, Hone T, et al. 2 .

BF - Bolsa Família; ESF - Estratégia Saúde da Família. 
Os resultados sugerem que os níveis de mortalidade de crianças de até 5 anos no Brasil tendem a ser substancialmente diferentes para cada cenário de gastos públicos, contrastando o cenário de redução da cobertura de programas de combate à pobreza e cuidados primários e a manutenção dos níveis de proteção social para atendimento das populações de maior vulnerabilidade. As estimativas indicam que, no cenário de manutenção da proteção social, a Taxa de Mortalidade de Crianças de até 5 anos (TMCA5) seria $8,6 \%$ mais baixa que a encontrada no cenário de austeridade, com um impacto de aproximadamente $20 \mathrm{mil}$ mortes evitadas de crianças até 5 anos entre 2017 e 2030. A TMCA5 de doenças diarreicas e subnutrição seria $39,3 \%$ e $35,8 \%$ mais baixas, respectivamente, e haveria 123 mil hospitalizações de crianças até 5 anos com a manutenção da proteção social. Segundo as estimativas do estudo, a redução nas coberturas de BF e ESF teriam impactos desproporcionalmente mais elevados em municípios mais pobres, contribuindo ainda mais para as desigualdades na saúde e comprometendo os esforços para alcance do terceiro e do décimo Objetivos de Desenvolvimento Sustentável (ODS).

De acordo com os autores, as estimativas de mortalidade mais elevada em decorrência de diarreia e subnutrição no cenário de austeridade são consistentes com a avaliação retrospectiva que atribuiu ao conjunto de programas BF e ESF um efeito mais importante na redução da mortalidade dessas causas que na mortalidade em geral16, além de estudos que indicam que essas doenças são fortemente associadas à pobreza ${ }^{17}$. Os impactos estimados de redução da cobertura de BF e ESF como medidas de austeridade podem ocorrer por meio de inúmeros mecanismos. Evidências sugerem que o BF melhora quantidade e qualidade da alimentação de famílias pobres ${ }^{8}$, acarretando melhor estado nutricional de crianças nas regiões mais pobres do Brasil9: e provavelmente explicam reduções na TMCA5 ${ }^{16,18}$. O programa BF também melhora condições de saúde da criança diretamente mediante promoção de cuidados pré-natal para gestantes, cobertura de vacinação e acompanhamentos de rotina como condicionantes para o recebimento do benefício.

Acerca da ESF, os dados sugerem que a expansão do programa reduz a mortalidade, promovendo acesso a cuidados pré-natal e ampliando a cobertura de vacinação ${ }^{18,19}$. Ademais, o programa amplia a disponibilidade de atividades médicas básicas e visitas domiciliares na área de cobertura ${ }^{19}$, bem como reduz desigualdades, com impacto ainda maior em municípios com Índice de Desenvolvimento Humano mais baixos ${ }^{\mathbf{1 8}}$. 
Figura 1. As taxas médicas de mortalidade infantil de menores de cinco anos (por mil nascidos vivos) para causas selecionadas do período 2010-2030 no cenário de crise econômica intermediária e duas respostas políticas (austeridade e manutenção da proteção social)
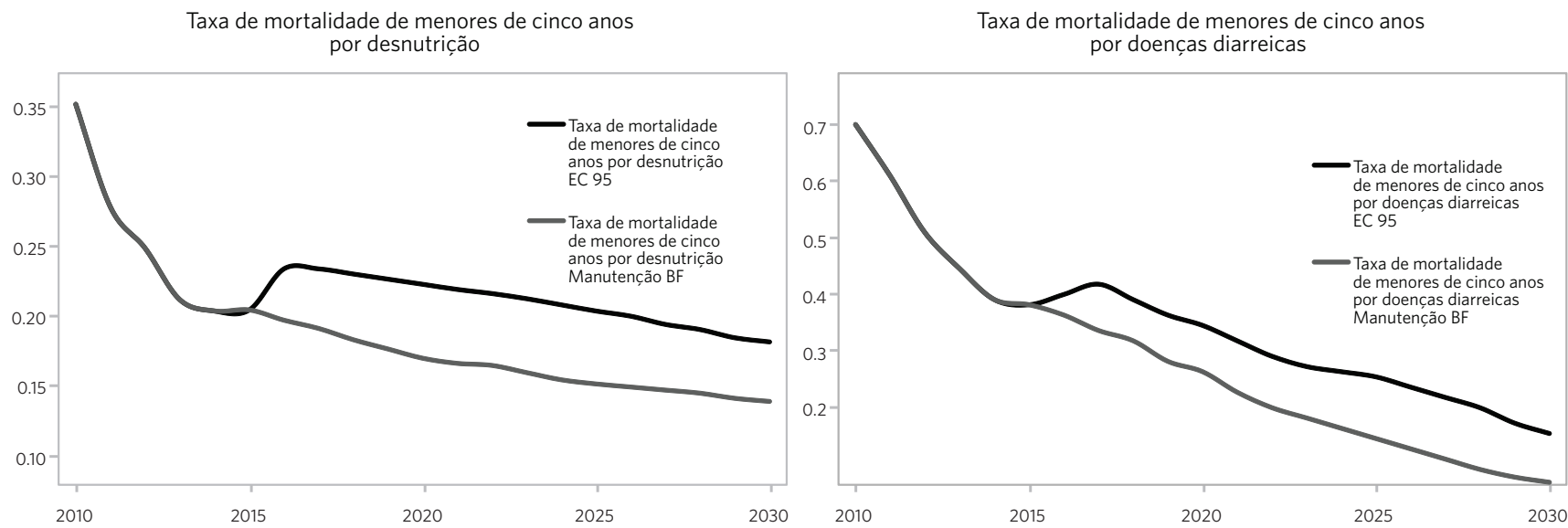

Fonte: Rasella D, Basu S, Hone T, et al.2.

BF - Bolsa Família; ESF - Estratégia Saúde da Família.

Foi implementado, no Brasil, um conjunto de políticas sociais ousadas com o intuito de reduzir a pobreza e alcançar cobertura de saúde universal ao longo dos últimos 20 anos $^{\mathbf{2 0 , 2 1}}$, sendo dois terços da população hoje coberta pelo ESF, e um

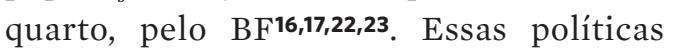
contribuíram para melhorias importantes nos indicadores e reduziram desigualdades na saúde ${ }^{21}$. Os resultados encontrados por Rasella et al. ${ }^{2}$ sugerem que a crise econômica atual, aliada a um pacote de medidas de austeridade, pode comprometer ainda mais os ganhos em redução de pobreza e melhoria dos indicadores de saúde, especialmente entre os mais pobres. O constante monitoramento dos impactos em pobreza e saúde das políticas de austeridade no Brasil e em qual medida essas restrições comprometem o alcance de ODS terão grande valor para agentes públicos mundo afora.

\section{Conclusão: a reemergência das 'mortes severinas'}

Em um dos poemas mais conhecidos da literatura brasileira, João Cabral de Melo Neto indica uma tipologia própria do morrer no agreste pernambucano. A morte severina

que é a morte que se morre de velhice antes dos trinta, de emboscada antes dos vinte, de fome um pouco por dia, (de fraqueza e de doença $[. .$.$] em qualquer idade, e até gente não$ nascida)24(204).

Em um tratamento menos literário, haveríamos de incluir entre as 'mortes severinas' as infecções associadas à desnutrição. Afinal, para os menores de 5 anos, por exemplo, infeções e desnutrição estão fortemente associadas. Para esse grupo etário, 50\% de todos os óbitos são devidos a cinco doenças infeciosas que estão usualmente associadas à 
desnutrição25. Esta produz imunodeficiência, enquanto a infeção está associada à má nutrição e às alterações nutricionais ${ }^{25}$. Embora o País não tenha tido sucesso na redução da mortalidade por causas violentas, os avanços nas políticas públicas de saúde e de assistência social contribuíram decisivamente para a prevalência residual dos óbitos por desnutrição e doenças diarreicas em crianças.

Desde o final do primeiro semestre de 2018, os jornais informam a piora de indicadores básicos de saúde. Há relatos de situações como o crescimento dos óbitos infantis ${ }^{26}$, o surto de 822 casos de sarampo registrados até 25 de julho de $2018^{27}$, a preocupação do Ministério da Saúde com o alto risco de retorno da pólio em pelo menos 312 cidades brasileiras ${ }^{27}$, a redução da cobertura vacinal de sarampo que apresentou redução de $95,4 \%$ para $83,9 \%$ no período de 2016 a $2018^{28}$. A piora desses indicadores sugere que a redução de investimentos em saúde se faz sentir na atenção básica, afetando a oferta de serviços de promoção, prevenção e atenção. É particularmente preocupante porque o Brasil tem grande domínio da tecnologia de vacinas, na qual é uma das grandes referencias no mundo. Vacinar é muito mais simples do que controlar vetores, como, por exemplo, combater os mosquitos transmissores de dengue, Chikungunya, Zika e malária.

Esses episódios recentes indicam os primeiros efeitos de um significativo agravamento do quadro de saúde no País, em especial para a população de baixa renda. O debate econômico atual apresenta erroneamente as medidas de austeridade fiscal como único caminho para um regime de responsabilidade fiscal. É interessante que o Brasil observe o que Portugal e a Espanha estão fazendo, após um ciclo desgastante de austeridade e piora do bem-estar social. Portugal, que refez sua rota de desenvolvimento há mais tempo, apresenta a melhora tanto dos seus indicadores econômicos como dos sociais. A Espanha passa a viver um novo momento de otimismo após anos de desesperança. O Brasil precisa rever sua rota de desenvolvimento não apenas para enfrentar os efeitos da crise econômica em curso, mas também para recuperar sua trajetória de inclusão social e econômica e de melhora da saúde de sua população.

\section{Colaboradores}

Paes-Sousa R contribuiu para elaboração do ensaio com as seguintes atividades: desenho, redação geral e edição final. Rasella D contribuiu para a revisão crítica do manuscrito. Carepa-Sousa J contribuiu para elaboração de versões preliminares do artigo e revisão de importante conteúdo intelectual. 


\section{Referências}

1. Massuda A, Hone T, Leles FA, et al. The Brazilian health system at crossroads: progress, crisis and resilience. BMJ Glob Health. 2018 jul; 3(4).

2. Rasella D, Basu S, Hone T, et al. Child morbidity and mortality associated with alternative policy responses to the economic crisis in Brazil: A nationwide microsimulation study. PLoS Medicine. 2018 maio 22; 15(5).

3. Fundação Getúlio Vargas. Boletim Macro IBRE/ FGV: Julho de 2018. Rio de Janeiro: FGV; 2018.

4. Vieira FS, Benevides RPDS. Os impactos do novo regime fiscal para o financiamento do Sistema Único de Saúde e para a efetivação do direito à saúde no Brasil. Brasília, DF: Instituto de Pesquisa Econômica Aplicada; 2016.

5. Schramm JMA, Paes-Sousa R, Mendes LVP. Políticas de austeridade e seus impactos na saúde: um debate em tempos de crise. Rio de Janeiro: Fiocruz; 2018.

6. Reeves A, McKee M, Basu S, et al. The political economy of austerity and healthcare: Cross-national analysis of expenditure changes in 27 European nations 1995-2011. Health policy. 2014 mar; 115(1):1-8.

7. Simou E, Koutsogeorgou E. Effects of the economic crisis on health and healthcare in Greece in the literature from 2009 to 2013: a systematic review. Health policy. 2014 abr; 115(2-3):111-119.

8. Grupo de Trabalho da Sociedade Civil para Agenda 2030. Relatório Luz da Agenda 2030 de Desenvolvimento Sustentável Síntese II. São Paulo: IDS; 2018.

9. Neri M. Qual foi o Impacto da Crise sobre Pobreza e Distribuição de Renda? Rio de Janeiro: FGV; 2018.

10. Doniec K, Dall'Alba R, King L. Brazil's health catastrophe in the making. The Lancet. 2018; 3(4).

11. Fernandes A, Rodrigues E. Gastos do governo fe- deral com saúde e educação caem 3,1\% em 2017 [internet]. O Estado de São Paulo. 2018 fev 6 [acesso em 2018 jul 30]. Disponível em: https://economia.estadao.com.br/noticias/geral,gastos-do-governo-federal-com-saude-e-educacao-caem-3-1-em-2017,70002179425.

12. Morosini MVGC, Fonseca AF, Lima LDD. Política Nacional de Atenção Básica 2017: retrocessos e riscos para o Sistema Único de Saúde. Saúde debate. 2018 jan-mar; 42(116):11-24.

13. Pains C. Brasil registra 822 casos de sarampo apenas este ano [internet]. O Globo. 2018 jul 31 [acesso em 2018 jul 30]. Disponível em: https://oglobo.globo.com/ saber-viver/brasil-registra-822-casos-de-sarampo-apenas-este-ano-22934212.

14. Paiva AB, Mesquita ACS, Jaccoud L, et al. O novo regime fiscal e suas implicações para a política de assistência social no Brasil. Brasília, DF: Instituto de Pesquisa Econômica Aplicada; 2016.

15. Rossi P, Dweck E. Impacts of the new fiscal regime on health and education. Cad Saúde Pública. 2016; 32:12-5.

16. Rasella D, Aquino R, Santos CAT, et al. Effect of a conditional cash transfer programme on childhood mortality: a nationwide analysis of Brazilian municipalities. Lancet. 2013; 382(9886):57-64.

17. Jones G, Steketee RW, Black RE, et al. How many child deaths can we prevent this year? Lancet. 2003; 362(9377):65-71.

18. Guanais FC. The combined effects of the expansion of primary health care and conditional cash transfers on infant mortality in Brazil, 1998-2010. Am J Public Health. 2013; 103(11):2000-2006.

19. Rasella D, Aquino R, Barreto ML. Reducing childhood mortality from diarrhea and lower respiratory tract infections in Brazil. Pediatrics. 2010; 126(3):534-540. 
20. Macinko J, Harris MJ. Brazil's family health strategy-delivering community-based primary care in a universal health system. N Engl J Med. 2015; 372(23):2177-2181.

21. Barreto ML, Rasella D, Machado DB, et al. Monitoring and evaluating progress towards universal health coverage in Brazil. PLoS Med. 2014; 11(9).

22. Brasil. Ministério da Saúde. Departamento de Atenção Básica. Histórico de cobertura. Brasília, DF: Ministério da Saúde; 2018.

23. Ministério do Desenvolvimento Social e Combate a Fome. Matriz de informação social. Rio de Janeiro: Ministério do Desenvolvimento Social e Combate a Fome; 2018.

24. Melo Neto, JC. Poesias completas: 1940-1965. 2. ed. Rio de Janeiro: J. Olympio. 1975.

25. Katona P, Katona-Apte J. The interaction between nutrition and infection. Clin Infect Dis. 2008; 46(10):1582-1588.
26. Collucci C, Merlo M, Pitombo JP. Com zika e crise no país, mortalidade infantil sobe pela $1^{\mathrm{a}}$ vez em 26 anos [internet]. Folha de São Paulo. 2018 jul 16 [acesso em 2018 jul 30]. Disponível em: https://folha.com/2j0oy6h9.

27. Modelli L. Sarampo, pólio, difteria e rubéola voltam a ameaçar após erradicação no Brasil [internet]. BBC Brasil. 2018 jul 7 [acesso em 2018 jul 30]. Disponível em: https://www.bbc.com/portuguese/ brasil-44706026.

28. Cancian N. Vacinação de crianças no país atinge índice mais baixo em 16 anos [internet]. Folha de São Paulo. 2018 jun 19 [acesso em 2018 jul 30]. Disponível em: https://folha.com/zfle20on.

Recebido em 05/08/2018

Aprovado em 09/09/2018

Conflito de interesses: inexistente

Suporte financeiro: não houve 